\title{
Inhaltsverzeichnis Jahrgang 1954
}

\section{Aufsätze}

Alt, A.: Eine neue Sammlung altorientalischer Texte zum Alten Testament . . . . . . . . EiBfeldt, 0.: Recht und Grenze archäologischer Betrachtung des Alten Testaments . . . . . . .

Gundert, W:: Neue Forschung zum japanischen Puppenspiele . . . . . . . . . . .

Hoffmann, K.: Bemerkungen zur vedischen Kosmologie

Oberhuber, K.: Zur Struktur des Sumerischen :

Werner, R.: Zur Stellung des Luwischen unter den altanatolischen Sprache

$$
\text { altanatolischen Sprachen }
$$

\section{Besprechungen}

Abel, F.-M.: Histoire de la Palestine depuis la conquête d'Alexandre jusqu'à l'invasion arabe. I. u. II. (O. EiBfeldt)

Aldred, C.: Old Kingdom Art in Ancient Egypt Middle Kingdom Art in Ancient Egypt - New Kingdom Art in Ancient Egypt (G. Roeder) . Alsdorf, L., s. Lüders, H.

Alt, A.: Kleine Schriften zur Geschichte des Volkes Israel. I. (J. Lind blom) . . . . . . .

Altan Tobèi: A brief History of the Mongols by bLobzaǹ bsTan-'jin. With a critical introduction by A. Mostaert ed. by F.W. Cleaves (E. Hae$\mathrm{nisch}$ )

Althelm, F.: Niedergang der Alten Welt. i. u. II. (W. Schubart)

Annual Blbliography of Indian Arehaeology. Vol. XV

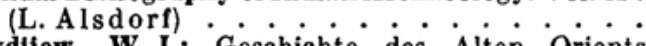

Awdijew, W. I.: Geschichte des Alten Orients

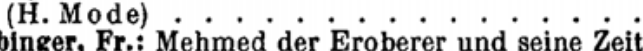

Babinger, Fr.: Mehmed der Eroberer und seine Zeit (Fr. Taeschner) . $\cdot \dot{R^{\prime}}$

Baramkl, D. C., s. Sellers, O. R.

Bardtke, H.: Die Handschriftenfunde am Toten Meer (C. K u hl) $\cdot{ }^{\circ} \cdot{ }^{\circ}$

Barnett, R. D., s. Woolley,

Beckerath, J. v.: Tanis und Theben (H. Kees) .

Benl, 0.: Die Entwicklung der japanischen Poetik bis zum 16. Jahrhundert (W. Gundert)

Bertholet, A.: Wörterbuch der Religionen. In Verb. m. H. v. Campenhausen verfaßt (O. Eißfeld t).

Bibliographie Bouddhique XXI-XXIII. Publiée avec le concours du Centre National de la $\mathrm{Re}$ cherche Scientifique (F. Weller).

Blssing, Fr. W. v.: Der Tote vor dem Opfertiseh (S. Schott)

- Versuch zur Bestimmung der Grundbedeutung des Wortes NUTR für Gott im Altägyptischen (S. Morenz)

Blachère, R., et Marie Cecealdi: Exercices d'Arabe classique. IIe. Ed. (A. Dietrich) . . . . .

Böhl, F. M. Th.: De Psalmen. II. (F. M a a B) . .

Böhlig, A.: Ägypten und Byzanz bis zur arabischen Zeit (W. C. Till)

- Ein Lexikon der griech. Wörter im Koptischen - Die griech.-lat. Lehnwörter in den koptischen manichäischen Texten (W. C. Till). . . .

Boessneck, J.: Die Haustiere in Altägypten (L.

Keimer). . E. . . . . . . . . . .

Bottéro, J.: La religion babylonienne (Fr. Schollmeyer) . '. Oppenheim, M. v.

Spalte Branden, A. van den: Les Inscriptions thamoudéen- Spalte nes (W. Caskel) ......... 51

Brandensteln, W.: Atlantis (F. Dornseiff) : . 306

Brockelmann, C.: A bessinische Studien (E.W agner) 465

Brunton, P.: Die Philosophie der Wahrheit - tiefster Grund des Yoga (G. Mensching) . . .

- Die Weisheit des Uberselbst (G. Mensching).

Bünger, K., und H. Trimborn: Religiöse Bindungen in frühen und in orientalischen Rechten hrsg. (M. San-Nicold) . . . . . . .

Buhot, J.: Arts de la Chine (K. Finsterbusch) Campenhausen, H. v., s. Bertholet, A.

293 Casal, J. M.: Fouilles de Virampatnam-Arikamedu. Rapport de l'Inde et de l'Occident aux environs de l'ère chrétienne (J. Ph. Vogel) . . . . .

Caskel, W., s. Oppenheim, M. v.

Cecealdi, Marie, s. Blachère, R.

Černy, J., s. Gardiner-Peet.

Chakravarti, A., s. Tirukkural

Chakravarti, K. C.: Ancient Indian Culture and Ci-

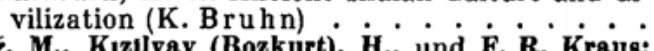

43 Eski Babil zamanına ait Nippur hukuki vesikalarl. Altbabylonische Rechtsurkunden aus Nippur (M. San Nicold). . . . . . . .

126 Cleaves, F. W., s. Altan Tobči.

Cölgecen, M. C.: Le Code d'Hammourabi (M. S a n Nicold) .

424 Conze, E.: Buddhism (Fr. Weller) . . . . . .

Curiel, R., und D. Schlumberger: Trésors monétaires d'Afghanistan (Hch. Junker) . . . . . . .

Dörner, F. K.: Bericht über eine Reise in Bithynien (Fr. Taeschner) . . . . . . . .

[Dornselff:] Festschrift Franz Dornseiff zum 65. Geburtstag, hrsg. v. H. Kusch (J. Leipoldt). .

Driver, G. R.: Aramaic Documents of the fifth Century B. C. transer. and ed. (O. Eißfeldt) ...

Dupont-Sommer, A.: Aperçus préliminaires sur les manuscrits de la Mer Morte (H. Bardtke) .

- Nouveaux Aperçus sur les manuscrits de la Mer Morte (H. Bardtke) . . . . . . . .

- Observations sur le manuel de discipline découvert près de la Mer Morte (H. Bardtke) . . .

337 Durr, J. A.: Deux traités grammaticaux tibétains (J. Schubert)

214 - Morphologie du verbe tibétain (J.Schubert).

Ebeling, E.: Bruchstücke einer mittelassyrischen Vorschriftensammlung für die Akklimatisierung und Trainierung von Wagenpferden (O. K rü $\mathrm{ck}$ mann) . .

Eberhard, W.: Die chinesische Novelle des 17.-19. Jahrhunderts. Eine soziologische Untersuchung (J. Prüšek). . . . . . . . . . . u. P. N. Boratav: Typen türkischer Volksmärchen (W. Ruben) . . . . . . . . . . . .

Elgood, P. G.: Later Dynasties of Egypt (G. Roeder) . . . . . . . . . . . . .

123 Endres, F. C.: Land der Träume (F. v. Rummel) .

Erman, A. $\dagger$, u. H. Grapow: Wörterbuch der ägyptischen Sprache. Die Belegstellen Bd. III (H. Kees) . . . . . . . . . . . .

- Das Wörterbuch der ägyptischen Sprache. Zur Geschichte eines großen wissenschaftlichen Unternehmens der A kademie (H. Bonnet) .

Falkenstein, A.: Grammatik der Sprache Gudeas von Lagaš. I. Schrift- und Formenlehre. II. Syn$\operatorname{tax}$ (E. Ebeling) . . . . . . . . . .

313 - u. W. v. Soden: Sumerische und akkadische Hymnen und Gebete (F. R. Kraus). . . .

Farrukh, 0. A.: 'abqarijat al-'arab fi'l-'ilm wa'l-falsafa. Le génie arabe dans la science et la philosophie. 20 ed. (A. Schimmel)....... 